\title{
Left Eigenvector of a Stochastic Matrix
}

\author{
Sylvain Lavallée \\ Departement de mathematiques, Universite du Quebec a Montreal, Montreal, Canada \\ E-mail:sylvain.lavallee@uqtr.ca \\ Received January 7, 2011; revised June 7, 2011; accepted June 15, 2011
}

\begin{abstract}
We determine the left eigenvector of a stochastic matrix $\boldsymbol{M}$ associated to the eigenvalue 1 in the commutative and the noncommutative cases. In the commutative case, we see that the eigenvector associated to the eigenvalue 0 is $\left(N_{1}, \cdots, N_{n}\right)$, where $N_{i}$ is the $i$-th principal minor of $\boldsymbol{N}=\boldsymbol{M}-\boldsymbol{I}_{n}$, where $\boldsymbol{I}_{n}$ is the identity matrix of dimension $n$. In the noncommutative case, this eigenvector is $\left(P_{1}^{-1}, \cdots, P_{n}^{-1}\right)$, where $P_{i}$ is the sum in $\mathbb{Q}\left\langle\left\langle a_{i j}\right\rangle\right\rangle$ of the corresponding labels of nonempty paths starting from $i$ and not passing through $i$ in the complete directed graph associated to $\boldsymbol{M}$.
\end{abstract}

Keywords: Generic Stochastic Noncommutative Matrix, Commutative Matrix, Left Eigenvector Associated To The Eigenvalue 1, Skew Field, Automata

\section{Introduction}

It is well known that 1 is one of the eigenvalue of a stochastic matrix (i.e. the sum of the elements of each row is equal to 1) and its associated right eigenvector is the vector $(1,1, \cdots, 1)^{T}$. But, what is the left eigenvector associated to 1 ?

In the commutative case, this eigenvector is the vector $\left(M_{1}, \cdots, M_{n}\right)$, where $M_{i}$ is the $i-t h$ principal minor of the matrix. This formula is known in probability theory; it amounts to finding the stationary distribution of the finite Markov chain whose transition matrix is the stochastic irreducible matrix.

In the noncommutative case, we must involve inverses of elements of the skew field and as these may be undefined, we take a generic noncommutative stochastic matrix: this is the matrix $\left(a_{i j}\right)$ of noncommuting variables $a_{i j}$ subject only to the stochastic identities; i.e. the sum of each row equals one.

We work in the free field generated by these variables (in the sense of Paul Cohn), which we call the stochastic free field. Considering the complete digraph on the set $\{1, \cdots, n\}$, let $M_{i}$ be the set of paths from $i$ to $i$. Let $P_{i}$ be the paths starting from $i$ and not passing through $i$ again. We identify $P_{i}$ with the noncommutative power series which is equal to the sum of all the words in $P_{i}$ and we still denote this series by $P_{i}$. Next we show that the elements $P_{i}^{-1}$ can be evaluated in the stochastic free field and that the vector

$\left(P_{1}^{-1}, \cdots, P_{n}^{-1}\right)$ is fixed by our matrix; moreover, the sum of the $P_{i}^{-1}$ is equal to 1 , hence they form a kind of noncommutative limiting probability.

These results have been proved in [1] but the proof proposed in this paper are completely different.

Indeed for the major part the proof in these two instances, we use elementary operations on the rows and the columns of the matrix.

\section{Commutative Case}

The commutative case is well known in probability theory. Indeed, we calculate the limit probability of a finite Markov chain by replacing the stochastic matrix $\boldsymbol{M}$ by $\boldsymbol{M}-\boldsymbol{I}$, where $\boldsymbol{I}$ is the identity matrix of the appropriate dimension. For this, we use the Markov chain tree Theorem, where this calculus is expressed in terms of spanning trees. The Markov chain tree theorem gives a formula for the stationary distribution of a finite Markov chain. Equivalently, this formula gives a row vector fixed by a matrix fixing $(1,1, \cdots, 1)^{T}$. This theorem is attributed to Kirchoff by Persi Diaconis, who gives a probabilistic proof of it (see [2] p. 443 and 444). See also $[3,4]$.

The proof uses only the definition of the determinant which involves the principal minors.

Proposition 1 Let $\boldsymbol{M}$ be a stochastic matrix and 
$\boldsymbol{N}=\boldsymbol{M}-\boldsymbol{I}_{n}=\left(b_{i j}\right)_{1 \leq i, j \leq n}$; i.e. for all $i=1, \cdots, n$,

$\sum_{j=1}^{n} b_{i j}=0$. Then $\left(N_{1}, \cdots, N_{n}\right)$, where $N_{i}$ is the $i-t h$ principal minor of $\boldsymbol{N}$, is the left eigenvector associated to the eigenvalue 0 .
Proof. By definition of $\boldsymbol{N}$, we have $\operatorname{det}(\boldsymbol{N})=0$. We show that

$$
\left(N_{1}, N_{2}, \cdots, N_{n}\right) \boldsymbol{N}=0
$$

We verify this Equation with the first column of $N$.

$$
\begin{aligned}
& N_{1} b_{11}+\cdots+N_{n} b_{n 1} \\
& =\left|\begin{array}{cccc}
b_{22} & b_{23} & & b_{2 n} \\
b_{32} & b_{33} & & b_{3 n} \\
\vdots & \vdots & \ddots & \vdots \\
b_{n 2} & b_{n 3} & & b_{n n}
\end{array}\right| b_{11}+\cdots+\left|\begin{array}{cccc}
b_{11} & b_{12} & & b_{1, n-1} \\
b_{21} & b_{22} & & b_{2, n-1} \\
\vdots & \vdots & \ddots & \vdots \\
b_{n-1,1} & b_{n-1,2} & & b_{n-1, n-1}
\end{array}\right| b_{n 1} \\
& =\left|\begin{array}{cccc}
b_{22} & b_{23} & & b_{2 n} \\
b_{32} & b_{33} & & b_{3 n} \\
\vdots & \vdots & \ddots & \vdots \\
b_{n 2} & b_{n 3} & & b_{n n}
\end{array}\right| b_{11}+\cdots+\left|\begin{array}{cccc}
-\sum_{j=2}^{n} b_{1 j} & b_{12} & & b_{1, n-1} \\
-\sum_{j=2}^{n} b_{2 j} & b_{22} & & b_{2, n-1} \\
\vdots & \vdots & \ddots & \vdots \\
-\sum_{j=2}^{n} b_{n-1, j} & b_{n-1,2} & & b_{n-1, n-1}
\end{array}\right| b_{n 1} \\
& =\left|\begin{array}{cccc}
b_{22} & b_{23} & & b_{2 n} \\
b_{32} & b_{33} & & b_{3 n} \\
\vdots & \vdots & \ddots & \vdots \\
b_{n 2} & b_{n 3} & & b_{n n}
\end{array}\right| b_{11}+\cdots-\underbrace{\left|\begin{array}{cccc}
b_{12} & b_{12} & & b_{1, n-1} \\
b_{22} & b_{22} & & b_{2, n-1} \\
\vdots & \vdots & \ddots & \vdots \\
b_{n-1,2} & b_{n-1,2} & & b_{n-1, n-1}
\end{array}\right|}_{=0} b_{n 1}-\cdots \\
& \underbrace{\left|\begin{array}{cccc}
b_{1, n-1} & b_{12} & & b_{1, n-1} \\
b_{2, n-1} & b_{22} & & b_{2, n-1} \\
\vdots & \vdots & \ddots & \vdots \\
b_{n-1, n-1} & b_{n-1,2} & & b_{n-1, n-1}
\end{array}\right|}_{=0} b_{n 1}-\left|\begin{array}{cccc}
b_{1 n} & b_{12} & & b_{1, n-1} \\
b_{2 n} & b_{22} & & b_{2, n-1} \\
\vdots & \vdots & \ddots & \vdots \\
b_{n-1, n} & b_{n-1,2} & & b_{n-1, n-1}
\end{array}\right| b_{n 1} \\
& =\left|\begin{array}{cccc}
b_{22} & b_{23} & & b_{2 n} \\
b_{32} & b_{33} & & b_{3 n} \\
\vdots & \vdots & \ddots & \vdots \\
b_{n 2} & b_{n 3} & & b_{n n}
\end{array}\right| b_{11}-\cdots-\left|\begin{array}{cccc}
b_{1 n} & b_{12} & & b_{1, n-1} \\
b_{2 n} & b_{22} & & b_{2, n-1} \\
\vdots & \vdots & \ddots & \vdots \\
b_{n-1, n} & b_{n-1,2} & & b_{n-1, n-1}
\end{array}\right| b_{n 1} \\
& =\left|\begin{array}{cccc}
b_{22} & b_{23} & & b_{2 n} \\
b_{32} & b_{33} & & b_{3 n} \\
\vdots & \vdots & \ddots & \vdots \\
b_{n 2} & b_{n 3} & & b_{n n}
\end{array}\right| b_{11}-\cdots-(-1)^{n-2}\left|\begin{array}{cccc}
b_{12} & b_{13} & & b_{1 n} \\
b_{22} & b_{23} & & b_{2 n} \\
\vdots & \vdots & \ddots & \vdots \\
b_{n-1,2} & b_{n-1,3} & & b_{n-1, n}
\end{array}\right| b_{n 1} \\
& =\left|\begin{array}{cccc}
b_{22} & b_{23} & & b_{2 n} \\
b_{32} & b_{33} & & b_{3 n} \\
\vdots & \vdots & \ddots & \vdots \\
b_{n 2} & b_{n 3} & & b_{n n}
\end{array}\right| b_{11}-\cdots+(-1)^{n+1}\left|\begin{array}{cccc}
b_{12} & b_{13} & & b_{1 n} \\
b_{22} & b_{23} & & b_{2 n} \\
\vdots & \vdots & \ddots & \vdots \\
b_{n-1,2} & b_{n-1,3} & & b_{n-1, n}
\end{array}\right| b_{n 1} \\
& =\operatorname{det}(\boldsymbol{N})=0
\end{aligned}
$$

Remark 2 If $\boldsymbol{M}$ is reducible, then this proposition is asserting that the zero vector ia an eigenvector. If $\boldsymbol{M}$ is irreducible and stochastic, then its adjoint will be of the form $(1, \cdots, 1) \boldsymbol{u}^{T}$, where $\boldsymbol{u}^{T}$ is a null vector. Te pro- 
position follows from the cofactor formula for the adjoint.

\section{Noncommutative Case}

In [1], the authors have prove in two manners that are actually similar, Theorem 9. In the first, results from variable-length prefix codes are required; and the second deals with general variable-length codes, not necessarily prefix. Moreover, in Appendix 2 of [1], we see how the theory of quasideterminants may be used to obtain these results on noncommutative matrices.

The major part of the new proof of this Theorem involves only the elementary operations on the rows and on the columns of the stochastic matrix. Therefore, we need the following results.

\subsection{Languages and Series}

Let $A$ be a finite alphabet and $A^{*}$ be the free monoid generated by $A$. A language is a subset of a free monoid $A^{*}$. A language is rational if it is obtained from finite languages by the operations (called rational) union, product (concatenation) and star. The product of two languages $L_{1} L_{2}$ is $\left\{w_{1} w_{2} \mid w_{1} \in L_{1}, w_{2} \in L_{2}\right\}$, and the star of $L \quad$ is $L^{*}=\left\{w_{1} \cdots w_{n} \mid w_{i} \in L, n \geq 0\right\}=\bigcup_{n \geq 0} L^{n}$. Rational languages may be obtained by using only unambiguous rational operations; these are: disjoint union, unambiguous product (meaning that if $w \in L_{1} L_{2}$, then $w$ has a unique factorization $w=w_{1} w_{2}, w_{i} \in L_{i}$ ) and the star $L^{*}$ restricted to languages which are free submonoids of $A^{*}$.

A formal series is an element of the $\mathbb{Q}$-algebra of noncommutative series $\mathbb{Q}\langle\langle A\rangle\rangle$, where $A$ is a set of noncommuting variables. A rational series is an element of the smallest subalgebra of $\mathbb{Q}\langle\langle A\rangle\rangle$, which contains the $\mathbb{Q}$-algebra of noncommutative polynomials $\mathbb{Q}\langle A\rangle$, and which is closed under the operation

$$
S \mapsto S^{*}=\sum_{n=0}^{\infty} S^{n}=(1-S)^{-1}
$$

which is defined if $S$ has zero constant term. We denote by $\mathbb{Q}\langle\langle A\rangle\rangle^{\text {rat }}$ the $\mathbb{Q}$-algebra of rational series.

Let $L$ be a rational language. Since $L$ may be obtained by unambiguous rational expressions, it follows that its characteristic series $\sum_{w \in L} w \in \mathbb{Q}\langle\langle A\rangle\rangle$ is rational. We shall identify a language and its characteristic series. This is exposed in [5] or [6].

\subsection{Free Field}

Let $\mathbb{K}$ be a field. If the multiplicative group of $\mathbb{K}$ is commutative, then $\mathbb{K}$ is a commutative field. Else, $\mathbb{K}$ is called skew field. The ring of rational formal series in noncommutative variables $\mathbb{Q}^{\text {rat }}\langle\langle A\rangle\rangle$ is not a skew field. However, skew fields containing $\mathbb{Q}\langle A\rangle$ do exist. One of them is the free field (in the sense of Cohn), denoted $\mathcal{F}$. The free field is the noncommutative analogue of the field of fractions of the ring of commutative polynomials. There are several constructions of the free field: Amitsur [7], Bergman [8], Malcolmson [9], Cohn [10] and [11].

A square matrix of order $n$ is full if it can't expressed as a product of matrices $n \times n-1$ by $n-1 \times n$.

Theorem 3 ([11], Thm 4.5.8.) Each full matrix $\boldsymbol{M}$ with coefficients in $\mathbb{K}\langle X\rangle$ is invertible in the free field.

A square matrix of order $n$ is called hollow if it contains a $p \times q$ block of zeros such that $p+q \geq n+1$.

Example 4 The matrix $\left[\begin{array}{lll}0 & 0 & 1 \\ 0 & 0 & 1 \\ 1 & 1 & 1\end{array}\right]$ is hollow since

there is a $2 \times 2$ block of 0 and $4>3$.

Proposition 5 ([11], Prop. 4.5.4) A hollow matrix is not full.

Proposition 6 Take $\lambda \in \mathcal{D}^{1 \times n}, \quad \boldsymbol{M} \in \mathcal{D}^{n \times n}$ and $\gamma \in \mathcal{D}^{n \times 1}$, where $\mathcal{D}$ is a skew field. We suppose that $\boldsymbol{M}$ is invertible. Then $\left[\begin{array}{cc}\boldsymbol{M} & \gamma \\ \lambda & 0\end{array}\right]$ is invertible if and only if $\lambda \boldsymbol{M}^{-1} \gamma \neq 0$.

Proof.

$(\Leftarrow)$ Suppose that $c=\lambda M^{-1} \gamma \neq 0$, then the inverse of $\left[\begin{array}{cc}\boldsymbol{M} & \gamma \\ \boldsymbol{\lambda} & 0\end{array}\right]$ is

$$
\left[\begin{array}{cc}
\boldsymbol{M}^{-1}-\boldsymbol{M}^{-1} \gamma c^{-1} \lambda M^{-1} & M^{-1} \gamma c^{-1} \\
c^{-1} \lambda M^{-1} & -c^{-1}
\end{array}\right]
$$

Indeed, this matrix is the right inverse of $\boldsymbol{M}$ since

$$
\begin{aligned}
& {\left[\begin{array}{ll}
\boldsymbol{M} & \gamma \\
\lambda & 0
\end{array}\right]\left[\begin{array}{cc}
\boldsymbol{M}^{-1}-\boldsymbol{M}^{-1} \gamma c^{-1} \lambda M^{-1} & \boldsymbol{M}^{-1} \gamma c^{-1} \\
c^{-1} \lambda M^{-1} & -c^{-1}
\end{array}\right]} \\
& =\left[\begin{array}{cc}
\boldsymbol{M}\left(\boldsymbol{M}^{-1}-\boldsymbol{M}^{-1} \gamma c^{-1} \lambda M^{-1}\right)+\gamma c^{-1} \lambda M^{-1} & \boldsymbol{M}\left(\boldsymbol{M}^{-1} \gamma c^{-1}\right)-\gamma c^{-1} \\
\lambda\left(\boldsymbol{M}^{-1}-\boldsymbol{M}^{-1} \gamma c^{-1} \lambda M^{-1}\right) & \lambda \boldsymbol{M}^{-1} \gamma c^{-1}
\end{array}\right] \\
& =\left[\begin{array}{cc}
1-\gamma c^{-1} \lambda M^{-1}+\gamma c^{-1} \lambda M^{-1} & \gamma c^{-1}-\gamma c^{-1} \\
\lambda M^{-1}-\underbrace{\lambda M^{-1} \gamma c^{-1}}_{=c} \lambda M^{-1} & \underbrace{\gamma M^{-1} \gamma c^{-1}}_{=c}
\end{array}\right]=\left[\begin{array}{ll}
1 & 0 \\
0 & 1
\end{array}\right]
\end{aligned}
$$


This matrix is also the left inverse of $\boldsymbol{M}$ since

$$
\begin{aligned}
& {\left[\begin{array}{cc}
M^{-1}-M^{-1} \gamma c^{-1} \lambda M^{-1} & M^{-1} \gamma c^{-1} \\
c^{-1} \lambda M^{-1} & -c^{-1}
\end{array}\right]\left[\begin{array}{cc}
M & \gamma \\
\lambda & 0
\end{array}\right]} \\
& =\left[\begin{array}{cc}
\left(M^{-1}-M^{-1} \gamma c^{-1} \lambda M^{-1}\right) M+M^{-1} \gamma c^{-1} \lambda & \left(M^{-1}-M^{-1} \gamma c^{-1} \lambda M^{-1}\right) \gamma \\
\left(c^{-1} \lambda M^{-1}\right) M-c^{-1} \lambda & \left(c^{-1} \lambda M^{-1}\right) \gamma
\end{array}\right] \\
& =\left[\begin{array}{cc}
1-M^{-1} \gamma c^{-1} \lambda+M^{-1} \gamma c^{-1} \lambda & M^{-1} \gamma-M^{-1} \gamma c^{-1} \underbrace{\lambda M^{-1} \gamma}_{=c} \\
c^{-1} \lambda-c^{-1} \lambda & c^{-1} \underbrace{\lambda M^{-1} \gamma}_{=c}
\end{array}\right]=\left[\begin{array}{ll}
1 & 0 \\
0 & 1
\end{array}\right]
\end{aligned}
$$

$(\Rightarrow)$ Suppose that $\left[\begin{array}{cc}\boldsymbol{M} & \gamma \\ \lambda & 0\end{array}\right]$ is invertible. Let

$\left[\begin{array}{cc}M^{\prime} & \gamma^{\prime} \\ \lambda^{\prime} & \alpha\end{array}\right]$ be its inverse. Then we have

$$
\left[\begin{array}{cc}
\boldsymbol{M} & \gamma \\
\boldsymbol{\lambda} & 0
\end{array}\right]\left[\begin{array}{l}
\boldsymbol{\gamma}^{\prime} \\
\boldsymbol{\alpha}
\end{array}\right]=\left[\begin{array}{c}
0 \\
\vdots \\
0 \\
1
\end{array}\right]
$$

Hence $\boldsymbol{M} \boldsymbol{\gamma}^{\prime}+\boldsymbol{\gamma} \boldsymbol{\alpha}=\left[\begin{array}{c}0 \\ \vdots \\ 0\end{array}\right]=0$ and $\lambda \boldsymbol{\gamma}^{\prime}=1$. It follows that

$$
\begin{aligned}
& \gamma^{\prime}=-M^{-1} \gamma \alpha \\
& \Rightarrow \lambda \gamma^{\prime}=-\lambda M^{-1} \gamma \alpha=1 \\
& \Rightarrow \lambda M^{-1} \gamma \neq 0
\end{aligned}
$$

From this proposition, we deduce the following result.

Corollary 7 Let $\lambda, \boldsymbol{M}$ and $\gamma$ be matrices with

coefficients in $\mathbb{K}\langle A\rangle$ where $\boldsymbol{M}$ is full. If $\left[\begin{array}{cc}\boldsymbol{M} & \gamma \\ \lambda & 0\end{array}\right]$ is not full (in particular, if $\left[\begin{array}{cc}\boldsymbol{M} & \gamma \\ \boldsymbol{\lambda} & 0\end{array}\right]$ is equivalent by elementary operations on the lines and on the columns to

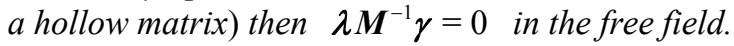

\subsection{Generic Stochastic Matrices}

Let $\boldsymbol{M}=\left(a_{i j}\right)_{1 \leq i, j \leq n}$ be a generic noncommutative matrix; i.e. the $a_{i j}$ are noncommutative variables. We denote $\mathcal{F}$ the corresponding free field. We associate to $\boldsymbol{M}$ the matrix $S$ : it's exactly the same matrix of which the coefficients satisfy the stochastic identities

$$
\forall i=1, \cdots, n, \sum_{j=1}^{n} a_{i j}=1
$$

In other words, the sum of each line of $S$ is equal to 1; hence $\boldsymbol{S}$ is a stochastic matrix. We call $\boldsymbol{S}$ a generic noncommutative stochastic matrix. The algebra over $\mathbb{Q}$ generated by its coefficients is a free associative algebra, since it is isomorph to the algebra $\mathbb{Q}\left\langle a_{i j}, i \neq j\right\rangle$. Indeed, we can eliminate the $a_{i i}$ with the stochastic relations (1). We denote this algebra by $\mathbb{Q}\left\langle a_{i j} /(1)\right\rangle$. Hence, there is a corresponding free field called stochastic free field denoted $\boldsymbol{S}$.

\subsection{Paths}

Consider the set of nonempty paths in the complete directed graph with a set of vertices $\{1, \cdots, n\}$ starting from $i$ and not passing through $i$; we denote by $P_{i}$ the sum in $\mathbb{Q}\left\langle\left\langle a_{i j}\right\rangle\right\rangle$ of all the corresponding words. It is classically a rational series, and thus defines an element of the free field $\mathcal{F}$.

$$
\text { Example } 8 \quad \boldsymbol{M}=\left[\begin{array}{ll}
a & b \\
c & d
\end{array}\right] \text {. The graph is }
$$

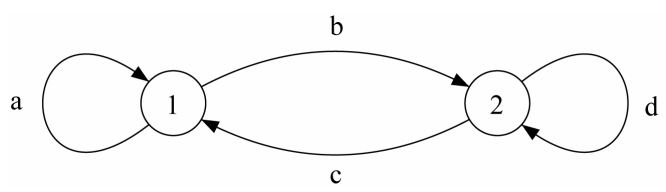

then

$$
P_{1}=1+b d^{*}, \quad P_{2}=1+c a^{*}
$$

We shall also consider rational expressions over any skew field $D$, and say that such an expression is evaluable in $D$ if it can be evaluated without inversion of 0 . If the elements of $D$ appearing in the rational expression are actually in a subring $R$ of $D$, we say that the expression is over $R$.

There is a canonical embedding of $\mathbb{Q}\langle\langle A\rangle\rangle^{r a t}$ into $\mathcal{F}$, which can be seen as follows: let $S$ be any rational series, we replace in it the operation $T^{*}$ by $(1-T)^{-1}$; then one obtains a rational expression in $\mathcal{F}$, which is evaluable in $\mathcal{F}$ and represents the image of $\boldsymbol{S}$ under the embedding $\mathbb{Q}\langle\langle A\rangle\rangle^{r a t} \longmapsto \mathcal{F}$. Thus, each rational 
language and each rational series is naturally an element of the free field. See [12].

By Theorem 1 of [1], the $P_{i}$ can be evaluated in the stochastic free field.

We can now state the main result of this paper.

Theorem 9 Let $\boldsymbol{M}=\left(a_{i j}\right)_{1 \leq i, j \leq n}$ be a generic stochastic noncommutative matrix. Let $P_{i}$ be defined as above. Then

$$
\left(P_{1}^{-1}, \cdots, P_{n}^{-1}\right) \boldsymbol{M}=\left(P_{1}^{-1}, \cdots, P_{n}^{-1}\right)
$$

In other words, $\left(P_{1}^{-1}, \cdots, P_{n}^{-1}\right)$ is the left eigenvector associated to the eigenvalue 1 of $\boldsymbol{M}$.

Example 10 (Continued.) Let be $S$ be the matrix $\boldsymbol{M}$ with $a+b=1$ and $c+d=1$. We show that

$$
\left(P_{1}^{-1}, P_{2}^{-1}\right) \boldsymbol{M}=\left(P_{1}^{-1}, P_{2}^{-1}\right)
$$

From this system, we deduce the two following equations:

$$
\begin{aligned}
& P_{1}^{-1} a+P_{2}^{-1}(1-d)=P_{1}^{-1} \\
& P_{1}^{-1}(1-a)+P_{2}^{-1} d=P_{2}^{-1}
\end{aligned}
$$

From the Equation (3), we have

$$
\begin{aligned}
& P_{1}^{-1} a+P_{2}^{-1}(1-d)=P_{1}^{-1} \\
& \Leftrightarrow P_{2}^{-1}(1-d)=P_{1}^{-1}(1-a) \\
& \Leftrightarrow d^{*} P_{2}=a^{*} P_{1} \\
& \Leftrightarrow d^{*}\left(1+c a^{*}\right)=a^{*}\left(1+b d^{*}\right) \\
& \Leftrightarrow d^{*}+d^{*}(1-d) a^{*}=a^{*}+a^{*}(1-a) d^{*} \\
& \Leftrightarrow d^{*}+a^{*}=a^{*}+d^{*}
\end{aligned}
$$

Hence, Equation (3) is satisfied. From the Equation (4), we have

$$
\begin{aligned}
& P_{1}^{-1}(1-a)+P_{2}^{-1} d=P_{2}^{-1} \\
& \Leftrightarrow P_{1}^{-1}(1-a)=\lambda_{2}^{-1}(1-d) \\
& \Leftrightarrow a^{*} P_{1}=d^{*} P_{2}
\end{aligned}
$$

Since we again obtain Equation (5), it follows that Equation (4) is also satisfied.

Proof of Theorem 9.

Let $\boldsymbol{M}=\left(a_{i j}\right)_{1 \leq i, j \leq n}$ be the matrix of the automaton $\mathcal{A}$ and $P_{i}$ be the language of the labels of the paths starting from $i$ and not passing through $i$. We have

$$
P_{i}=\sum_{j=1}^{n} L_{i j}, \quad i=1, \cdots, n,
$$

where

$$
L_{i j}=\left\{\begin{array}{cl}
1, & \text { if } i=j \\
\sum_{k=1}^{n} L_{i k} a_{k j}=a_{i j}+\sum_{k=1, k \neq j}^{n} L_{i k} a_{k j}, & \text { if } i \neq j
\end{array}\right.
$$

Let $\left(S_{i}\right)$ be the system composed of the $n$ Equations $P_{i}, L_{i 1}, \cdots, L_{i, i-1}, L_{i, i+1}, \cdots, L_{i n}$. Multiplying to the left by $P_{i}^{-1}$ each equation of $S_{i}$, we obtain the system

$$
\left(T_{i}\right):\left\{\begin{array}{l}
1=P_{i}^{-1}+\sum_{j=1, j \neq i}^{n} P_{i}^{-1} L_{i j}, \quad i=1, \cdots, n \\
P_{i}^{-1} L_{i j}=P_{i}^{-1} a_{i j}+\sum_{k=1, k \neq i}^{n} P_{i}^{-1} L_{i k} a_{k j}
\end{array}\right.
$$

Let $Q_{i j}=P_{i}^{-1} L_{i j}$, then we have

$$
\left(T_{i}\right):\left\{\begin{array}{l}
1=P_{i}^{-1}+\sum_{j=1, j \neq i}^{n} Q_{i j}, \quad i=1, \cdots, n \\
Q_{i j}=P_{i}^{-1} a_{i j}+\sum_{k=1, k \neq i}^{n} Q_{i k} a_{k j}
\end{array}\right.
$$

This system transforms into the following system

$$
\left(U_{i}\right):\left\{\begin{array}{l}
1=P_{i}^{-1}+\sum_{j=1, j \neq i}^{n} Q_{i j}, \quad i=1, \cdots, n \\
0=P_{i}^{-1} a_{i j}+Q_{i j}\left(a_{j j}-1\right)+\sum_{\substack{k=1, k \neq i, k \neq j}}^{n} Q_{i k} a_{k j}
\end{array}\right.
$$

We show that

$$
\sum_{k=1}^{n} P_{k}^{-1} a_{k 1} \equiv P_{1}^{-1}
$$

Define

$$
R=\sum_{k=1}^{n} P_{k}^{-1} a_{k 1}-P_{1}^{-1}
$$

We show that $R=0$. So Equation (6) is converted into the following Equation

$$
R-P_{1}^{-1}\left(1-a_{11}\right)-\sum_{k=2}^{n} P_{k}^{-1} a_{k 1}=0
$$

Consider the system of the $n^{2}+1$ Equations: Equation (7) and the $n$ systems $\left(U_{1}\right), \cdots,\left(U_{n}\right)$. We have the matrical representation

$$
\begin{aligned}
& \left(R, P_{1}^{-1}, Q_{12}, Q_{13}, \cdots, Q_{1 n}, P_{2}^{-1}, Q_{21}, Q_{23}, \cdots, Q_{2 n},\right. \\
& \left.P_{n}^{-1}, Q_{n 1}, \cdots, Q_{n, n-1}\right) \cdot \boldsymbol{E}=\lambda
\end{aligned}
$$




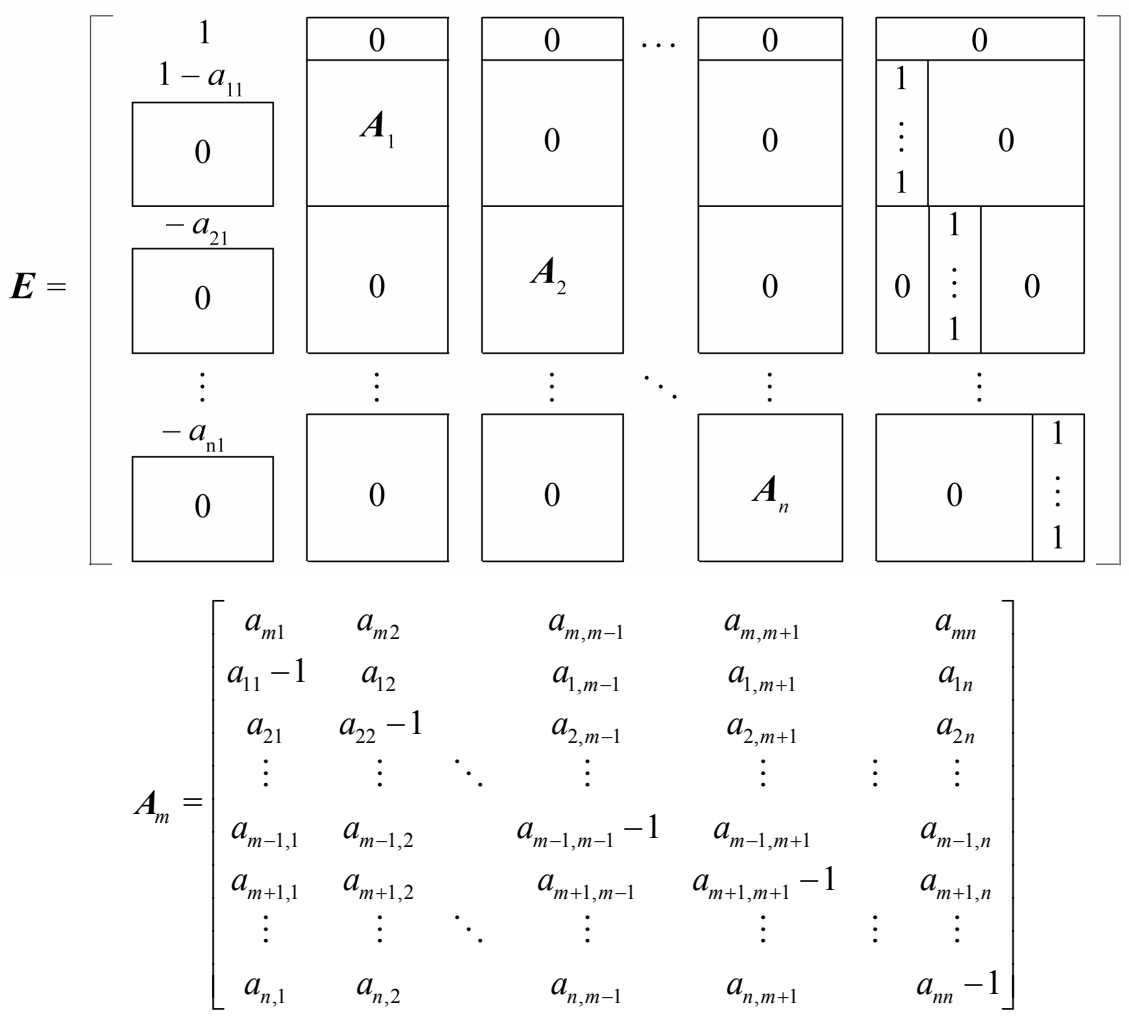

where $m=1, \cdots, n$ and $\lambda=(0, \cdots, 0, \underbrace{1, \cdots, 1}_{n \text { times }}) . \quad \boldsymbol{E}$ is a square matrix of order $n^{2}+1$. We have $\boldsymbol{R}=\boldsymbol{\lambda} \boldsymbol{E}^{-1} \boldsymbol{\gamma}$, $\gamma=(1, \underbrace{0, \cdots, 0}_{n^{2} \text { times }})^{T}$ and $\boldsymbol{F}=\left[\begin{array}{cc}\boldsymbol{E} & \gamma \\ \boldsymbol{\lambda} & 0\end{array}\right]$. The order of this matrix is $n^{2}+2$. Moreover, with the stochastic identities, we have
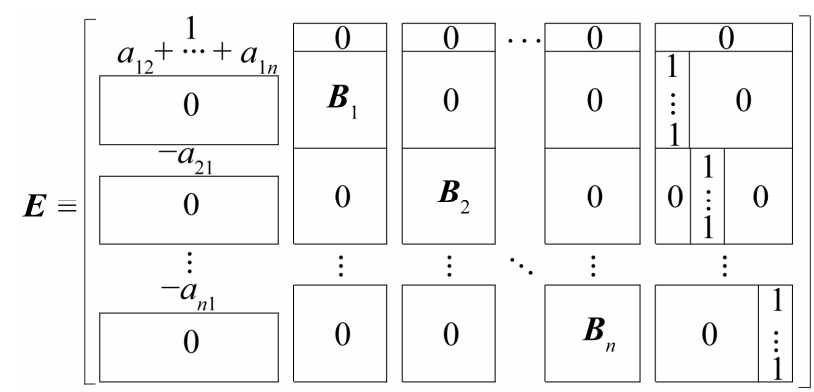
where the $\boldsymbol{B}_{i}$ are defined by

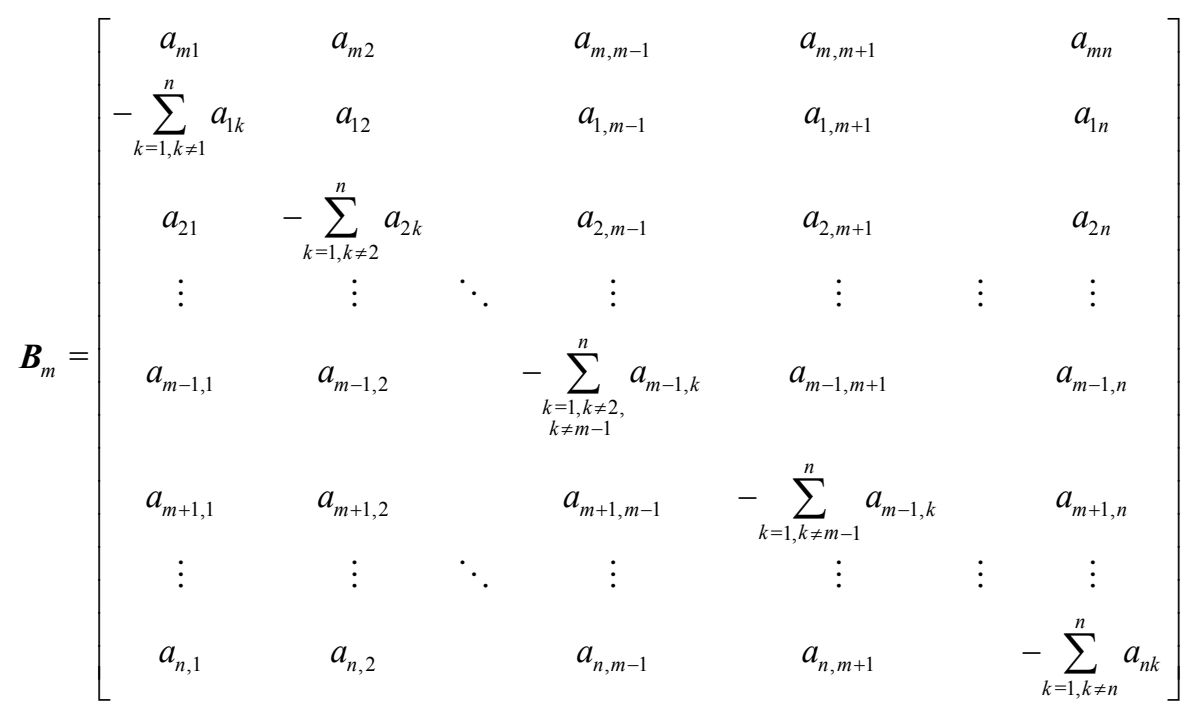


To show that $\boldsymbol{R}=0$, we show that the matrix $\boldsymbol{F}$ is hollow. For this, we apply elementary operations on the rows and on the columns of $\boldsymbol{F}$ until $\boldsymbol{F}$ contains a $s \times t$ block of 0 such that $s+t \geq n^{2}+3$.

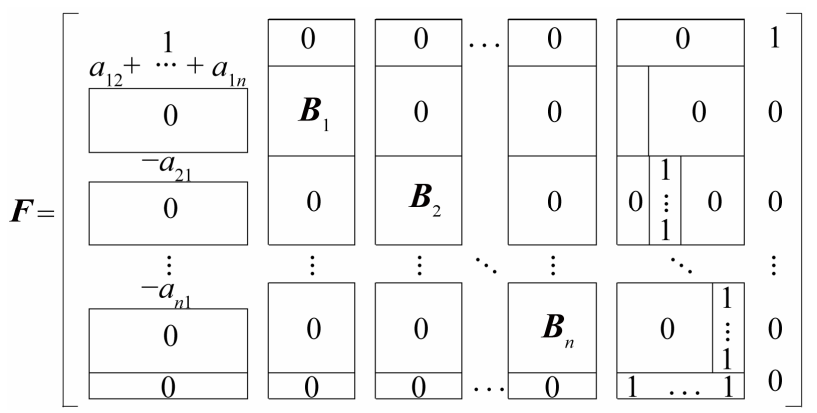

Step 1. We eliminate the first rowe and the last column of $\boldsymbol{F}$. We obtain the square matrix $\boldsymbol{F}_{1}$ of order $n^{2}+1\left(s+t \geq n^{2}+2\right)$.

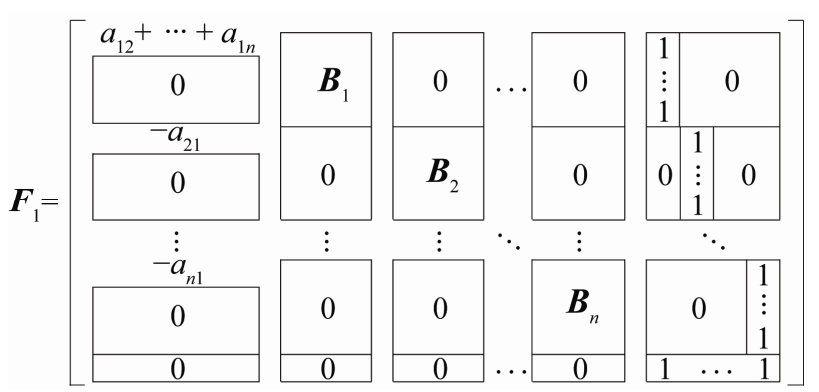

$$
\boldsymbol{C}_{m}=\left[\begin{array}{cc}
-\sum_{k=1, k \neq 1}^{n} a_{1 k} & a_{12} \\
a_{21} & -\sum_{k=1, k \neq 2}^{n} a_{2 k} \\
\vdots & \vdots \\
a_{n, 1} & a_{n, 2}
\end{array}\right.
$$

$m=2, \cdots, n$. We reduce $\boldsymbol{F}_{2}$ in removing the last line and the $n$-th column if we start from the end. We have the following matrix $\boldsymbol{F}_{3}$ of order $n^{2}$,

$(s+t \geq n+1)$.

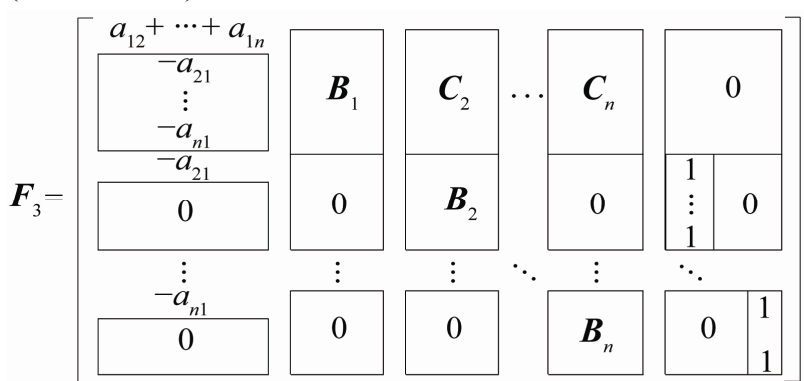

Step 3. Consider the rows $L_{i}$ of $\boldsymbol{F}_{3}$, where $i>n$ and $i \neq 0 \bmod n$. We want that the $(n-1)$ last elements of these rows to be equals to 0 . Let $i=k n+m$, where $0 \leq m \leq n-1$, then we apply the transformation
Step 2. We want a $n \times n$ block of 0 in the right upper corner of $F_{1}$. For this, we consider the $j$-th row of $F_{1}$ (corresponding to the $j-t h$ row of $B_{1}$ ). By construction of $B_{1}$, the first index of the elements of the $j$-th row of this block is $j$. Now, we consider the row which passes in the block $B_{2}$ such that this first index of its elements is $j$. Such a row exists by construction of $B_{2}$. We repeat this operation with the row which have the first index equals to $j$ in the blocks $B_{3}, \cdots, B_{n}$. We add each one of these rows to the $j$-th row of $F_{1}$. It follows that the $n$ last elements of the $j$ first rows of this new matrix are all equal to 1 . From each row of the $j$ first rows, we subtract the last row of $F_{1}$. We obtain the following matrix

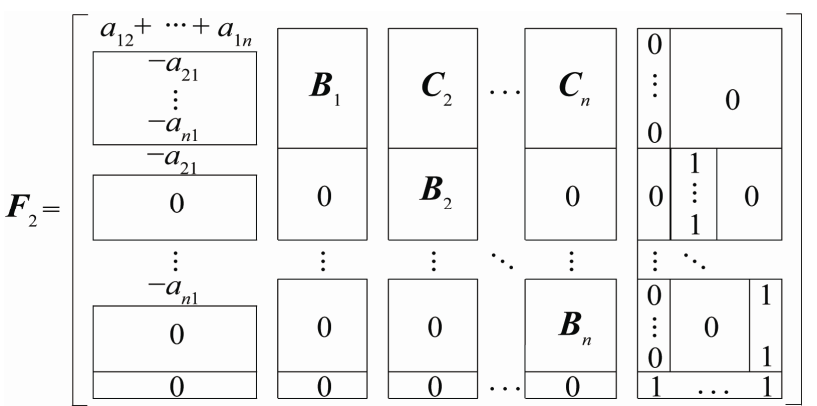

where

$$
\left.\begin{array}{cccc}
a_{1, m-1} & a_{1, m+1} & & a_{1 n} \\
a_{2, m-1} & a_{2, m+1} & & a_{2 n} \\
\vdots & \vdots & \vdots & \vdots \\
a_{n, m-1} & a_{n, m+1} & & -\sum_{k=1, k \neq n}^{n} a_{n k}
\end{array}\right]
$$

$L_{i}-L_{(k+1) n}=L_{k n+m}-L_{(k+1) n}$, to obtain the matrix

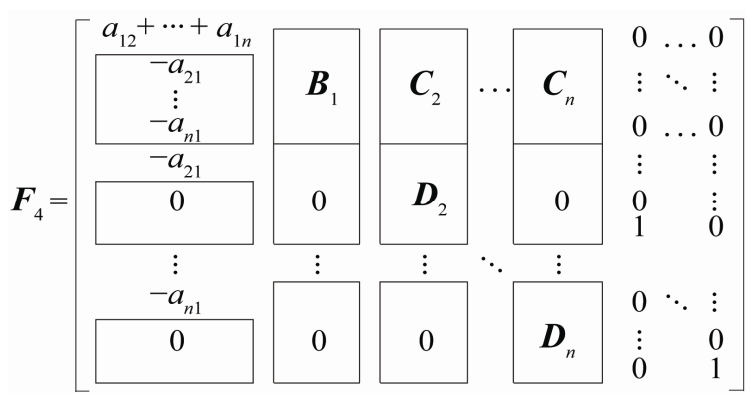

where, for all $m=2, \cdots, n$,

$$
\boldsymbol{D}_{m}=\left(d_{i j}^{m}\right)_{1 \leq i, j \leq n}, \boldsymbol{B}_{m}=\left(b_{i j}^{m}\right), d_{i j}=b_{i j}^{m}-b_{n j}^{m}
$$

We remove the $(n-1)$ rows $L_{i}$ where $i>n$ and $n \mid i$ and the $(n-1)$ last columns of $\boldsymbol{F}_{4}$ to obtain the matrix $\boldsymbol{F}_{5}$ of order $\left(s+t \geq n^{2}-n+2\right)$. 

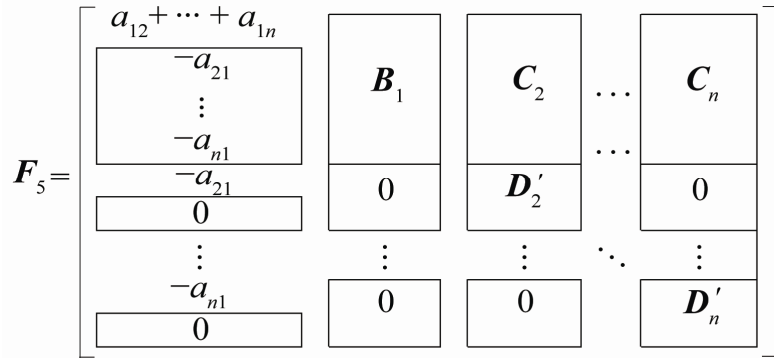

where $\boldsymbol{D}_{m}^{\prime}$ is the matrix obtained from $\boldsymbol{D}_{m}$ in removing the last row.

Step 4. Denote by $C_{m}^{1}$ the first column of the matrix $\boldsymbol{C}_{m}$ and by $d_{m}$ the column of $\boldsymbol{F}_{5}$, which is the extension of $\boldsymbol{C}_{m}^{1}$. We want $C_{m}^{1}$ to be transformed into a vector of $0, m=2, \cdots, n$. For this, we apply the operation $d_{j}+c_{2}+c_{3}++c_{n}$, where $c_{i}$ is the $i-t h$ column of $\boldsymbol{F}_{3}$.

Finally we want that the first $n$ elements of the first column of $\boldsymbol{F}_{5}$ to be all 0 ; for this, we apply the transformation $c_{1}-c_{2}--c_{n}$. We obtain the matrix $\boldsymbol{F}_{6}$ of order $n^{2}-n+1 \quad\left(s+t \geq n^{2}-n+2\right)$

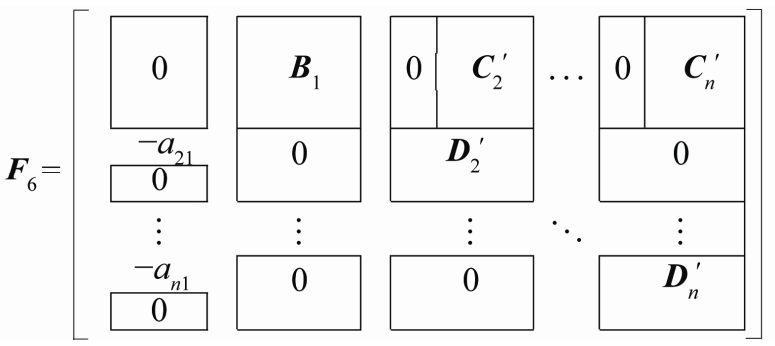

where $\boldsymbol{C}_{m}^{\prime}$ is the matrix obtained from $\boldsymbol{C}_{m}$ by removing the first column.

Step 5. We want each $\boldsymbol{C}_{m}^{\prime}$ will be transformed into a matrix of 0 . To each column $q$ of $\boldsymbol{C}_{m}^{\prime}$, denoted $\left(\boldsymbol{C}_{m}^{\prime}\right)^{q}$ corresponding to the column $d_{q}=\left(\left(\boldsymbol{C}_{m}^{\prime}\right)^{q}, *, \cdots, *\right)^{T}$ of $\boldsymbol{F}_{6}$. By construction of $\boldsymbol{C}_{m}^{\prime}$ (hence of $C_{m}^{\prime}$ ), there exists a column $b_{q}$ of $\boldsymbol{F}_{6}$ such that $b_{q}=\left(\left(\boldsymbol{C}_{m}^{\prime}\right)^{q}, *, \cdots, *\right)^{T}$. Hence, for all $q$, we calculate $d_{q}-b_{q}$. We obtain the matrix $\boldsymbol{F}_{7}$ of order $n^{2}-n+1\left(s+t \geq n^{2}-n+2\right)$.

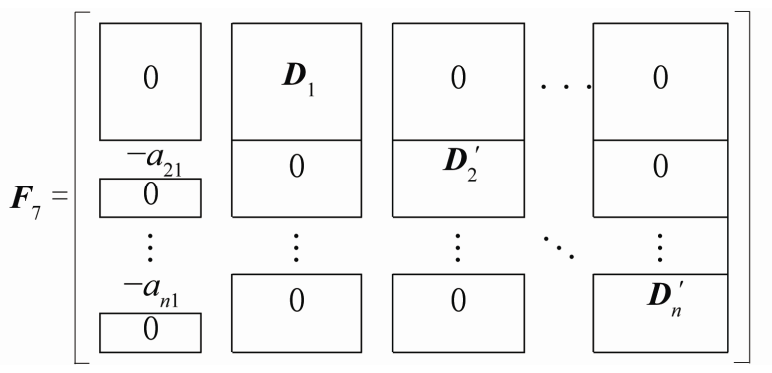

Step 6. Permuting the first and the $n-t h$ column of $\boldsymbol{F}_{7}$, we obtain the matrix $\boldsymbol{F}_{8}$ of order $n^{2}-n+1$ $\left(s+t \geq n^{2}-n+2\right)$.

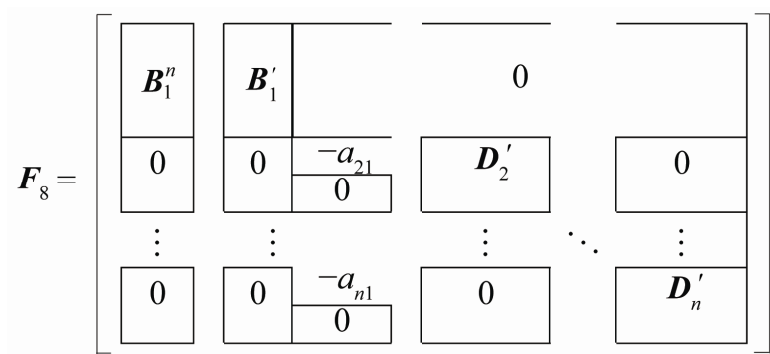

where $\boldsymbol{B}_{1}^{\prime}$ is the block obtained from $\boldsymbol{B}_{1}$ by removing the first column. We find a $n \times(n-1)^{2}+1$ block of 0 located in the right upper corner and

$$
n+(n-1)^{2}+1=n^{2}-n+2 \geq n^{2}-n+1=\operatorname{dim}\left(\boldsymbol{F}_{8}\right)
$$

It follows that $\boldsymbol{F}$ is a hollow. From Corollary 7 , $\boldsymbol{R}=\boldsymbol{\lambda} \boldsymbol{E}^{-1} \boldsymbol{\gamma}=\sum_{k=1}^{n} P_{k}^{-1} a_{k 1}-P_{1}^{-1}=0$. Hence $\left(P_{1}^{-1}, \cdots, P_{n}^{-1}\right)$ is the left eigenvector associated to 1 .

Example 11. Let $\boldsymbol{M}=\left[\begin{array}{lll}a_{11} & a_{12} & a_{13} \\ a_{21} & a_{22} & a_{23} \\ a_{31} & a_{32} & a_{33}\end{array}\right]$ be a generic noncommutative matrix in which the variables satisfy the stochastic identities $a_{i 1}+a_{i 2}+a_{i 3}=1, i=1,2,3$. We associate the automaton

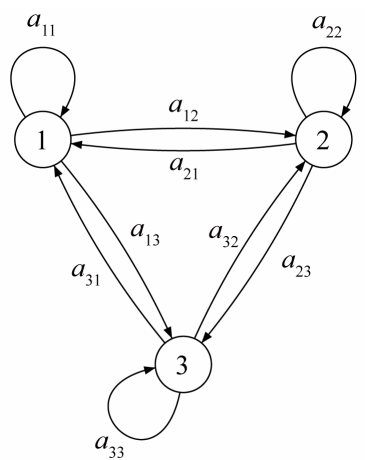

Let $P_{1}$ be the language recognized by the automaton

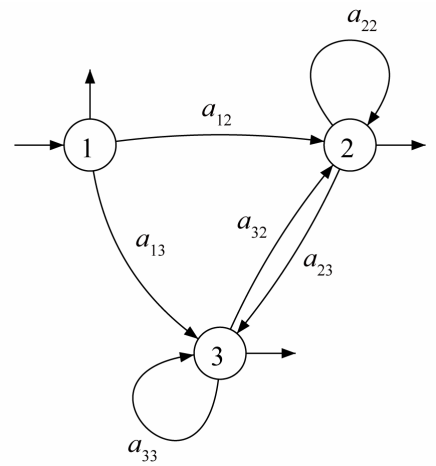

Let $L_{i j}$ be the language of words which start from $i$ to $j$. We have $P_{1}=L_{11}+L_{12}+L_{13}$, where

$$
L_{11}=1
$$




$$
\begin{aligned}
& L_{12}=a_{12}+L_{12} a_{22}+L_{13} a_{32} \\
& L_{13}=a_{13}+L_{12} a_{23}+L_{13} a_{33}
\end{aligned}
$$

We have the system

$$
\left(S_{1}\right):\left\{\begin{array}{l}
P=1+L_{12}+L_{13} \\
L_{12}=a_{12}+L_{12} a_{22}+L_{13} a_{32} \\
L_{13}=a_{13}+L_{12} a_{23}+L_{13} a_{33}
\end{array}\right.
$$

Multiplying to left each equation of $S_{1}$ by $P_{1}^{-1}$, we obtain the system

$$
\left(T_{1}\right):\left\{\begin{array}{l}
1=P_{1}^{-1}+P_{1}^{-1} L_{12}+P_{1}^{-1} L_{13} \\
P_{1}^{-1} L_{12}=P_{1}^{-1} a_{12}+P_{1}^{-1} L_{12} a_{22}+P_{1}^{-1} L_{13} a_{32} \\
P_{1}^{-1} L_{13}=P_{1}^{-1} a_{13}+P_{1}^{-1} L_{12} a_{23}+P_{1}^{-1} L_{13} a_{33}
\end{array}\right.
$$

Define $Q_{i j}=P_{1}^{-1} L_{i j}$, then

$$
T_{1}:\left\{\begin{array}{l}
1=P_{1}^{-1}+Q_{12}+Q_{13} \\
Q_{12}=P_{1}^{-1} a_{12}+Q_{12} a_{22}+Q_{13} a_{32} \\
Q_{13}=P_{1}^{-1} a_{13}+Q_{12} a_{23}+Q_{13} a_{33}
\end{array}\right.
$$

We obtain

$$
\left(U_{1}\right):\left\{\begin{array}{l}
1=P_{1}^{-1}+Q_{12}+Q_{13} \\
0=P_{1}^{-1} a_{12}+Q_{12}\left(a_{22}-1\right)+Q_{13} a_{32} \\
0=P_{1}^{-1} a_{13}+Q_{12} a_{23}+Q_{13}\left(a_{33}-1\right)
\end{array}\right.
$$$$
\boldsymbol{E}=\left[\begin{array}{cccccccccc}
1 & 0 & 0 & 0 & 0 & 0 & 0 & 0 & 0 & 0 \\
1-a_{11} & a_{12} & a_{13} & 0 & 0 & 0 & 0 & 1 & 0 & 0 \\
0 & a_{22}-1 & a_{23} & 0 & 0 & 0 & 0 & 1 & 0 & 0 \\
0 & a_{32} & a_{33}-1 & 0 & 0 & 0 & 0 & 1 & 0 & 0 \\
-a_{21} & 0 & 0 & a_{21} & a_{23} & 0 & 0 & 0 & 1 & 0 \\
0 & 0 & 0 & a_{11}-1 & a_{13} & 0 & 0 & 0 & 1 & 0 \\
0 & 0 & 0 & a_{31} & a_{33}-1 & 0 & 0 & 0 & 1 & 0 \\
-a_{31} & 0 & 0 & 0 & 0 & a_{31} & a_{32} & 0 & 0 & 1 \\
0 & 0 & 0 & 0 & 0 & a_{11}-1 & a_{12} & 0 & 0 & 1 \\
0 & 0 & 0 & 0 & 0 & a_{21} & a_{22}-1 & 0 & 0 & 1
\end{array}\right]
$$

We have $\boldsymbol{R}=\boldsymbol{\lambda} \boldsymbol{E}^{-1} \gamma$, where $\gamma=(1,0,0,0,0,0,0,0,0,0)^{T}$ and $E$ with stochastic iden-

$$
\left[\begin{array}{cccccccccc}
1 & 0 & 0 & 0 & 0 & 0 & 0 & 0 & 0 & 0 \\
a_{12}+a_{13} & a_{12} & a_{13} & 0 & 0 & 0 & 0 & 1 & 0 & 0 \\
0 & -a_{21}-a_{23} & a_{23} & 0 & 0 & 0 & 0 & 1 & 0 & 0 \\
0 & a_{32} & -a_{31}-a_{32} & 0 & 0 & 0 & 0 & 1 & 0 & 0 \\
-a_{21} & 0 & 0 & a_{21} & a_{23} & 0 & 0 & 0 & 1 & 0 \\
0 & 0 & 0 & -a_{12}-a_{13} & a_{13} & 0 & 0 & 0 & 1 & 0 \\
0 & 0 & 0 & a_{31} & -a_{31}-a_{32} & 0 & 0 & 0 & 1 & 0 \\
-a_{31} & 0 & 0 & 0 & 0 & a_{31} & a_{32} & 0 & 0 & 1 \\
0 & 0 & 0 & 0 & 0 & -a_{12}-a_{13} & a_{12} & 0 & 0 & 1 \\
0 & 0 & 0 & 0 & 0 & a_{21} & -a_{21}-a_{23} & 0 & 0 & 1
\end{array}\right]
$$
the two following systems

$$
\begin{aligned}
& \left(U_{2}\right):\left\{\begin{array}{l}
1=P_{2}^{-1}+Q_{21}+Q_{23} \\
0=P_{2}^{-1} a_{21}+Q_{12}\left(a_{11}-1\right)+Q_{23} a_{31} \\
0=P_{2}^{-1} a_{23}+Q_{21} a_{13}+Q_{23}\left(a_{33}-1\right)
\end{array}\right. \\
& \left(U_{3}\right):\left\{\begin{array}{l}
1=P_{3}^{-1}+Q_{31}+Q_{32} \\
0=P_{3}^{-1} a_{31}+Q_{31}\left(a_{11}-1\right)+Q_{32} a_{21} \\
0=P_{3}^{-1} a_{32}+Q_{31} a_{12}+Q_{32}\left(a_{22}-1\right)
\end{array}\right.
\end{aligned}
$$
suffices to prove that $P_{1}^{-1} a_{11}+P_{2}^{-1} a_{21}+P_{3}^{-1} a_{31}=P_{1}^{-1}$.

\section{Define}

then

$$
R=P_{1}^{-1} a_{11}+P_{2}^{-1} a_{21}+P_{3}^{-1} a_{31}-P_{1}^{-1}
$$

$$
R+P_{1}^{-1}\left(1-a_{11}\right)-P_{2}^{-1} a_{21}-P_{3}^{-1} a_{31}=0
$$

Under the matrical system, we have

$$
\left(R, P_{1}-1, Q_{12}, Q_{13}, P_{2}^{-1}, Q_{21}, Q_{23}, P_{3}^{-1}, Q_{31}, Q_{32}\right) \boldsymbol{E}=\boldsymbol{\lambda}
$$

where

$$
\lambda=(0,0,0,0,0,0,0,1,1,1)
$$

and

tities equal to

Permuting the index of the equations of $U_{1}$, we obtain

To show that $\left(P_{1}^{-1}, P_{2}^{-1}, P_{3}^{-1}\right) \boldsymbol{M}=\left(P_{1}^{-1}, P_{2}^{-1}, P_{3}^{-1}\right)$, it 
Let $\boldsymbol{F}=\left[\begin{array}{ll}\boldsymbol{E} & \gamma \\ \boldsymbol{\lambda} & 0\end{array}\right]$, we show that $\boldsymbol{F}$ is hollow.

$$
\boldsymbol{F}=\left[\begin{array}{ccccccccccc}
1 & 0 & 0 & 0 & 0 & 0 & 0 & 0 & 0 & 0 & 1 \\
a_{12}+a_{13} & a_{12} & a_{13} & 0 & 0 & 0 & 0 & 1 & 0 & 0 & 0 \\
0 & -a_{21}-a_{23} & a_{23} & 0 & 0 & 0 & 0 & 1 & 0 & 0 & 0 \\
0 & a_{32} & -a_{31}-a_{32} & 0 & 0 & 0 & 0 & 1 & 0 & 0 & 0 \\
-a_{21} & 0 & 0 & a_{21} & a_{23} & 0 & 0 & 0 & 1 & 0 & 0 \\
0 & 0 & 0 & -a_{12}-a_{13} & a_{13} & 0 & 0 & 0 & 1 & 0 & 0 \\
0 & 0 & 0 & a_{31} & -a_{31}-a_{32} & 0 & 0 & 0 & 1 & 0 & 0 \\
-a_{31} & 0 & 0 & 0 & 0 & a_{31} & a_{32} & 0 & 0 & 1 & 0 \\
0 & 0 & 0 & 0 & 0 & -a_{12}-a_{13} & a_{12} & 0 & 0 & 1 & 0 \\
0 & 0 & 0 & 0 & 0 & a_{21} & -a_{21}-a_{23} & 0 & 0 & 1 & 0 \\
0 & 0 & 0 & 0 & 0 & 0 & 0 & 1 & 1 & 1 & 0
\end{array}\right]
$$$$
\boldsymbol{F}_{1}=\left[\begin{array}{cccccccccc}
a_{12}+a_{13} & a_{12} & a_{13} & 0 & 0 & 0 & 0 & 1 & 0 & 0 \\
0 & -a_{21}-a_{23} & a_{23} & 0 & 0 & 0 & 0 & 1 & 0 & 0 \\
0 & a_{32} & -a_{31}-a_{32} & 0 & 0 & 0 & 0 & 1 & 0 & 0 \\
-a_{21} & 0 & 0 & a_{21} & a_{23} & 0 & 0 & 0 & 1 & 0 \\
0 & 0 & 0 & -a_{12}-a_{13} & a_{13} & 0 & 0 & 0 & 1 & 0 \\
0 & 0 & 0 & a_{31} & -a_{31}-a_{32} & 0 & 0 & 0 & 1 & 0 \\
-a_{31} & 0 & 0 & 0 & 0 & a_{31} & a_{32} & 0 & 0 & 1 \\
0 & 0 & 0 & 0 & 0 & -a_{12}-a_{13} & a_{12} & 0 & 0 & 1 \\
0 & 0 & 0 & 0 & 0 & a_{21} & -a_{21}-a_{23} & 0 & 0 & 1 \\
0 & 0 & 0 & 0 & 0 & 0 & 0 & 1 & 1 & 1
\end{array}\right]
$$

$L_{1}+L_{5}+L_{8}-L_{10}, \quad L_{2}+L_{4}+L_{9}-L_{10}, \quad L_{3}+L_{6}+L_{7}-L_{10}$,

$$
\boldsymbol{F}_{2}=\left[\begin{array}{cccccccccc}
a_{12}+a_{13} & a_{12} & a_{13} & -a_{12}-a_{13} & a_{13} & -a_{12}-a_{13} & a_{12} & 0 & 0 & 0 \\
-a_{21} & -a_{21}-a_{23} & a_{23} & a_{21} & a_{23} & a_{21} & -a_{21}-a_{23} & 0 & 0 & 0 \\
-a_{31} & a_{32} & -a_{31}-a_{32} & a_{31} & -a_{31}-a_{32} & a_{31} & a_{32} & 0 & 0 & 0 \\
-a_{21} & 0 & 0 & a_{21} & a_{23} & 0 & 0 & 0 & 1 & 0 \\
0 & 0 & 0 & -a_{12}-a_{13} & a_{13} & 0 & 0 & 0 & 1 & 0 \\
0 & 0 & 0 & a_{31} & -a_{31}-a_{32} & 0 & 0 & 0 & 1 & 0 \\
-a_{31} & 0 & 0 & 0 & 0 & a_{31} & a_{32} & 0 & 0 & 1 \\
0 & 0 & 0 & 0 & 0 & -a_{12}-a_{13} & a_{12} & 0 & 0 & 1 \\
0 & 0 & 0 & 0 & 0 & a_{21} & -a_{21}-a_{23} & 0 & 0 & 1 \\
0 & 0 & 0 & 0 & 0 & 0 & 0 & 1 & 1 & 1
\end{array}\right]
$$




$$
\boldsymbol{F}_{3}=\left[\begin{array}{ccccccccc}
a_{12}+a_{13} & a_{12} & a_{13} & -a_{12}-a_{13} & a_{13} & -a_{12}-a_{13} & a_{12} & 0 & 0 \\
-a_{21} & -a_{21}-a_{23} & a_{23} & a_{21} & a_{23} & a_{21} & -a_{21}-a_{23} & 0 & 0 \\
-a_{31} & a_{32} & -a_{31}-a_{32} & a_{31} & -a_{31}-a_{32} & a_{31} & a_{32} & 0 & 0 \\
-a_{21} & 0 & 0 & a_{21} & a_{23} & 0 & 0 & 1 & 0 \\
0 & 0 & 0 & -a_{12}-a_{13} & a_{13} & 0 & 0 & 1 & 0 \\
0 & 0 & 0 & a_{31} & -a_{31}-a_{32} & 0 & 0 & 1 & 0 \\
-a_{31} & 0 & 0 & 0 & 0 & a_{31} & a_{32} & 0 & 1 \\
0 & 0 & 0 & 0 & 0 & -a_{12}-a_{13} & a_{12} & 0 & 1 \\
0 & 0 & 0 & 0 & 0 & a_{21} & -a_{21}-a_{23} & 0 & 1
\end{array}\right]
$$

$L_{4}-L_{6}, \quad L_{5}-L_{6}, \quad L_{7}-L_{9}, \quad L_{8}-L_{9}$,

$$
\begin{aligned}
& \boldsymbol{F}_{4}=\left[\begin{array}{ccccccccc}
a_{12}+a_{13} & a_{12} & a_{13} & -a_{12}-a_{13} & a_{13} & -a_{12}-a_{13} & a_{12} & 0 & 0 \\
-a_{21} & -a_{21}-a_{23} & a_{23} & a_{21} & a_{23} & a_{21} & -a_{21}-a_{23} & 0 & 0 \\
-a_{31} & a_{32} & -a_{31}-a_{32} & a_{31} & -a_{31}-a_{32} & a_{31} & a_{32} & 0 & 0 \\
-a_{21} & 0 & 0 & a_{21}-a_{31} & a_{23}+a_{31}+a_{32} & 0 & 0 & 0 & 0 \\
0 & 0 & 0 & -a_{12}-a_{13}-a_{31} & a_{13}+a_{31}+a_{32} & 0 & 0 & 0 & 0 \\
0 & 0 & 0 & a_{31} & -a_{31}-a_{32} & 0 & 0 & 1 & 0 \\
-a_{31} & 0 & 0 & 0 & 0 & a_{31}-a_{21} & a_{32}+a_{21}+a_{23} & 0 & 0 \\
0 & 0 & 0 & 0 & 0 & -a_{12}-a_{13}-a_{21} & a_{12}+a_{21}+a_{23} & 0 & 0 \\
0 & 0 & 0 & 0 & 0 & a_{21} & -a_{21}-a_{23} & 0 & 1
\end{array}\right] \\
& \boldsymbol{F}_{5}=\left[\begin{array}{ccccccc}
a_{12}+a_{13} & a_{12} & a_{13} & -a_{12}-a_{13} & a_{13} & -a_{12}-a_{13} & a_{12} \\
-a_{21} & -a_{21}-a_{23} & a_{23} & a_{21} & a_{23} & a_{21} & -a_{21}-a_{23} \\
-a_{31} & a_{32} & -a_{31}-a_{32} & a_{31} & -a_{31}-a_{32} & a_{31} & a_{32} \\
-a_{21} & 0 & 0 & a_{21}-a_{31} & a_{23}+a_{31}+a_{32} & 0 & 0 \\
0 & 0 & 0 & -a_{12}-a_{13}-a_{31} & a_{13}+a_{31}+a_{32} & 0 & 0 \\
-a_{31} & 0 & 0 & 0 & 0 & a_{31}-a_{21} & a_{32}+a_{21}+a_{23} \\
0 & 0 & 0 & 0 & 0 & -a_{12}-a_{13}-a_{21} & a_{12}+a_{21}+a_{23}
\end{array}\right]
\end{aligned}
$$

$C_{1}-C_{2}-C_{3}, \quad C_{4}+C_{2}+C_{3}, \quad C_{6}+C_{2}+C_{3}$,

$$
\boldsymbol{F}_{6}=\left[\begin{array}{ccccccc}
0 & a_{12} & a_{13} & 0 & a_{13} & 0 & a_{12} \\
0 & -a_{21}-a_{23} & a_{23} & 0 & a_{23} & 0 & -a_{21}-a_{23} \\
0 & a_{32} & -a_{31}-a_{32} & 0 & -a_{31}-a_{32} & 0 & a_{32} \\
-a_{21} & 0 & 0 & a_{21}-a_{31} & a_{23}+a_{31}+a_{32} & 0 & 0 \\
0 & 0 & 0 & -a_{12}-a_{13}-a_{31} & a_{13}+a_{31}+a_{32} & 0 & 0 \\
-a_{31} & 0 & 0 & 0 & 0 & a_{31}-a_{21} & a_{32}+a_{21}+a_{23} \\
0 & 0 & 0 & 0 & 0 & -a_{12}-a_{13}-a_{21} & a_{12}+a_{21}+a_{23}
\end{array}\right.
$$


$C_{5}-C_{3}, \quad C_{7}-C_{2}$,

$$
\boldsymbol{F}_{7}=\left[\begin{array}{ccccccc}
0 & a_{12} & a_{13} & 0 & 0 & 0 & 0 \\
0 & -a_{21}-a_{23} & a_{23} & 0 & 0 & 0 & 0 \\
0 & a_{32} & -a_{31}-a_{32} & 0 & 0 & 0 & 0 \\
-a_{21} & 0 & 0 & a_{21}-a_{31} & a_{23}+a_{31}+a_{32} & 0 & 0 \\
0 & 0 & 0 & -a_{12}-a_{13}-a_{31} & a_{13}+a_{31}+a_{32} & 0 & 0 \\
-a_{31} & 0 & 0 & 0 & 0 & a_{31}-a_{21} & a_{32}+a_{21}+a_{23} \\
0 & 0 & 0 & 0 & 0 & -a_{12}-a_{13}-a_{21} & a_{12}+a_{21}+a_{23}
\end{array}\right]
$$

$C_{1} \leftrightarrow C_{3}$

$$
\boldsymbol{F}_{8}=\left[\begin{array}{ccccccc}
a_{13} & a_{12} & 0 & 0 & 0 & 0 & 0 \\
a_{23} & -a_{21}-a_{23} & 0 & 0 & 0 & 0 & 0 \\
-a_{31}-a_{32} & a_{32} & 0 & 0 & 0 & 0 & 0 \\
0 & 0 & -a_{21} & a_{21}-a_{31} & a_{23}+a_{31}+a_{32} & 0 & 0 \\
0 & 0 & 0 & -a_{12}-a_{13}-a_{31} & a_{13}+a_{31}+a_{32} & 0 & 0 \\
0 & 0 & -a_{31} & 0 & 0 & a_{31}-a_{21} & a_{32}+a_{21}+a_{23} \\
0 & 0 & 0 & 0 & 0 & -a_{12}-a_{13}-a_{21} & a_{12}+a_{21}+a_{23}
\end{array}\right.
$$

column. Again, we show that $\boldsymbol{F}^{\prime}$ is hollow, and hence,

We have a $3 \times 5$ block of zero with $8>7$. It follows that $\boldsymbol{F}$ is hollow. By Corollary 7 , $\boldsymbol{R}=P_{1}^{-1} a_{11}+P_{2}^{-1} a_{21}+P_{3}^{-1} a_{31}-P_{1}^{-1}=0$.

This proof allows us to prove the next result.

Theorem 12. Let $P_{i}$ be defined as above, then in $\mathcal{S}$ we have

$$
\sum_{i=1}^{n} P_{i}^{-1}=1
$$

Proof. Define

$$
\boldsymbol{R}^{\prime}=1-\sum_{i=1}^{n} P_{i}^{-1}
$$

we will show that

$$
\boldsymbol{R}^{\prime}+\sum_{i=1}^{n} P_{i}^{-1}=1
$$

We replace the first column of the matrix $\boldsymbol{E}$ by the vector

$$
(1,1,0, \cdots, 0,1,0, \cdots, 0, \cdots, 1,0, \cdots, 0)^{T}
$$

and the first element of $\lambda$ by 1 . Denoted $\boldsymbol{E}^{\prime}$ and $\lambda^{\prime}$ these two matrices. Consider the matrix $\boldsymbol{F}^{\prime}=\left[\begin{array}{ll}\boldsymbol{E}^{\prime} & \gamma \\ \lambda^{\prime} & 0\end{array}\right]$. We repeat each step of the proof of Theorem 9, except for the last operation of step 4 , which concerns the first by Corollary 7, we have $\boldsymbol{R}^{\prime}=0$. This proves Equation (8).

Example 13. (Continued.)

$$
\begin{aligned}
P_{1}^{-1}+P_{2}^{-1} & =P_{1}^{-1}+P_{1}^{-1}(1-a) d^{*} \quad \text { by Equation (5) } \\
& =P_{1}^{-1}+P_{1}^{-1} b d^{*}=P_{1}^{-1}\left(1+b d^{*}\right)=P_{1}^{-1} P_{1}=1
\end{aligned}
$$

\section{Conclusions}

One of the contribution of this article is the using of elementary operations on the lines and the columns. This method provides a new way to obtain some results in skew field theory with a minimum of knowledge of this theory. Moreover, Theorems 9 and 12 are proved exactly the same way.

\section{Acknowledgements}

I am obliged to thank to Christophe Reutenauer for his comments, corrections and suggestions.

\section{References}

[1] S. Lavall'ee, D. Perrin, C. Reutenauer and V. Retakh, "Codes and Noncommutative Stochastic Matrices," To 
appear, 2008.

[2] A. Broder, "Generating Random Spanning Trees. Proc 30th IEEE Symp," Proceedings of the 30th IEEE Symposium on Foundation of Computer Science, Boston, 1989, pp. 442-447.

[3] V. Anantharam and P. Tsoucas, "A Proof of the Markov Chain Tree Theorem," Statistic and Probability Letters, Vol. 8, No. 2, 1989, pp. 189-192.

[4] D.-J. Aldous, "The Randow Walk Construction of Uniform Spanning Trees and Uniform Labelled Trees," SIAM Journal on Discrete Mathematics, Vol. 3, No. 4, 1990, pp. 450-465.

[5] J. Berstel and C. Reutenauer, "Les S'eries Rationnelles et Leurs Langages," Masson, Paris, 1984.

[6] J. Berstel and C. Reutenauer, "Rational Series and Their Languages," 2008.

http://www-igm.univ-mlv.fr/ berstel/LivreSeries/LivreSe ries08janvier2008.pdf.
[7] A. Amitsur, "Rational Identities and Applications to Algebra and Geometry," Journal of Algebra, Vol. 3, 1966, pp. 304-359.

[8] G. M. Bergman, "Skew Field of Noncommutative Rational Functions, after Amitsur," S'eminaire Schu"tzenberger-Lentin-Nivat, Vol. 16, 1970.

[9] P. Malcolmson, "A Prime Matrix Ideal Yields a Skew Field," Journal of the London Mathematical Society, Vol. 18, 1978, pp. 221-233.

[10] P. M. Cohn, "Free Rings and Their Relations," Academic Press, Salt Lake City, 1971.

[11] P. M. Cohn, "Skew Fields: Theory of General Division Rings," Encyclopedia of Mathematics and Its Applications, Cambridge University Press, Cambridge, 1995.

[12] M. Fliess, "Sur le Plongement de l'alg ebre des S'eries Rationelles non Commutatives dans un Corps Gauche," Proceedings of the National Academy of Sciences, Paris, 1970. 